\title{
Physics of Supernova Neutrinos
}

\author{
Cecilia Lunardini* ${ }^{\dagger}$ \\ Institute for Advanced Study, Einstein Drive, Princeton 08540, USA \\ E-mail: ilunardi@ias.edui
}

\begin{abstract}
I review the effects of flavor conversion of neutrinos from stellar collapse due to masses and mixing, and discuss the motivations for their study. I consider in detail the sensitivity of certain observables (characteristics of the energy spectra of $\nu_{e}$ and $\bar{\nu}_{e}$ events) to the 13-mixing $\left(\sin ^{2} \theta_{13}\right)$ and to the type of mass hierarchy/ordering $\left(\operatorname{sign}\left[\Delta m_{13}^{2}\right]\right)$. These observables are: the ratio of average energies of the spectra, $r_{E} \equiv\langle E\rangle /\langle\bar{E}\rangle$, the ratio of widths of the energy distributions, $r_{\Gamma} \equiv \Gamma / \bar{\Gamma}$, the ratios of total numbers of $\nu_{e}$ and $\bar{\nu}_{e}$ events at low energies, $S$, and in the high energy tails, $R_{\text {tail }}$. I show that regions in the space of observables $r_{E}, r_{\Gamma}, R_{\text {tail }}$ exist in which certain mass hierarchy and intervals of $\sin ^{2} \theta_{13}$ can be identified or discriminated. Finally, I discuss the potential of studying regeneration effects on $\nu_{e}$ and $\bar{\nu}_{e}$ in the matter of the Earth and point out that both the observation or exclusion of these effects lead to model-independent information on $\sin ^{2} \theta_{13}$ and the mass hierarchy.
\end{abstract}

\section{Introduction and motivations}

The mechanism of neutrino flavor conversion due to masses and flavor mixing has been recently established by the combination of the results of solar neutrino detectors and those of the KamLand experiment iin. Results from the detection of atmospheric neutrinos and the preliminary data from the $\mathrm{K} 2 \mathrm{~K}$ experiment $\left[\begin{array}{l}2 \bar{i} \\ ]\end{array}\right]$ strongly support the existence of this phenomenon.

From the analysis of all the available data, we get a partial reconstruction of the neutrino masses $m_{i}$ (the label $i=1,2,3$ denotes the neutrino mass eigenstates) and of the mixing matrix $U$, defined by $\nu_{\alpha}=\sum_{i} U_{\alpha i} \nu_{i}$, where $\nu_{\alpha}(\alpha=e, \mu, \tau)$ are the flavor eigenstates. Using the standard parameterization of the mixing matrix in terms of three angles, $\theta_{12}, \theta_{13}, \theta_{23}$, we have:

$$
m_{2}^{2}-m_{1}^{2} \equiv \Delta m_{21}^{2}=(4-30) \cdot 10^{-5} \mathrm{eV}^{2}, \quad \tan ^{2} \theta_{12}=0.25-0.85
$$

${ }^{*}$ Speaker.

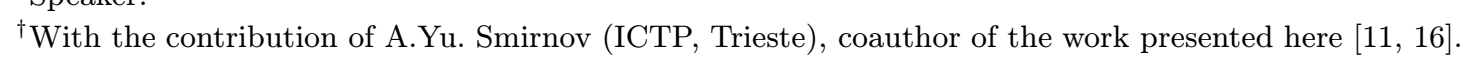


from solar neutrinos and KamLand, and

$$
m_{3}^{2}-m_{2}^{2} \equiv \Delta m_{32}^{2}= \pm(1.5-4) \cdot 10^{-3} \mathrm{eV}^{2}, \quad \tan ^{2} \theta_{23}=0.48-2.1
$$

from atmospheric neutrinos. The sign of $\Delta m_{32}^{2}$ is unknown. The two possibilities, $\Delta m_{32}^{2} \approx$ $\Delta m_{31}^{2}>0$ and $\Delta m_{32}^{2} \approx \Delta m_{31}^{2}<0$, are referred to as normal and inverted mass hierarchies/ordering respectively (abbreviated as n.h. and i.h. in the text).

The mixing angle $\theta_{13}$, which describes the $\nu_{e}$ content of the third mass eigenstate, $\nu_{3}$, is still unmeasured. We have an upper bound from the CHOOZ and Palo Verde experiments

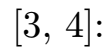

$$
\sin ^{2} \theta_{13} \lesssim 0.02
$$

The identification of the neutrino mass hierarchy and the determination of $\theta_{13}$ have become the main issues of further studies.

To achieve these, and other important goals, the study of neutrinos from core collapse supernovae is particularly interesting. Indeed, these neutrinos are produced and propagate in unique physical conditions of high density and high temperature, and therefore can manifest effects otherwise unaccessible. As will be discussed in the following, due to the very large interval of matter densities realized there, the interior of a collapsing star is the only environment where a neutrino of a given energy undergoes two MSW resonances, associated to the two mass squared splittings of the neutrino spectrum. This implies a richer phenomenology of flavor conversion, and therefore wider possibilities to probe the relevant parameters, with respect to the case of neutrinos in solar system, where only one resonance, i.e. one mass splitting, is relevant at a time.

It is important to consider, however, that the study of supernova neutrinos is not exempt of problems. The main obstacle is the absence of a "Standard Model" for supernova neutrinos, i.e. of precise predictions for the fluxes and energy spectra of neutrinos of different flavors originally produced in the star. The features of these fluxes depend on many details of the neutrino transport inside the star and, in general, on the type of

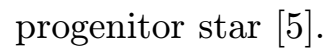

Since observables depend both on the features of the original fluxes and on the flavor conversion effects, it is clear that the extraction of information on the neutrino mixing and on the neutrino mass spectrum requires a careful consideration of astrophysical uncertainties.

\section{Properties of supernova neutrino fluxes and density profile of the star}

Neutrinos and antineutrinos of all the three flavors are produced in a supernova and emitted in a burst of $\sim 10$ seconds duration. At a given time $t$ from the core collapse the original flux of the neutrinos of a given flavor, $\nu_{\alpha}$, can be described by a "pinched" Fermi-Dirac (F-D) spectrum ${ }^{1}$,

$$
F_{\alpha}^{0}\left(E, T_{\alpha}, \eta_{\alpha}, L_{\alpha}, D\right)=\frac{L_{\alpha}}{4 \pi D^{2} T_{\alpha}^{4} F_{3}\left(\eta_{\alpha}\right)} \frac{E^{2}}{e^{E / T_{\alpha}-\eta_{\alpha}+1}},
$$

\footnotetext{
${ }^{1}$ An alternative parameterization has been recently suggested in $[\overline{5} 5]$.
} 
where $D$ is the distance to the supernova (typically $D \sim 10 \mathrm{kpc}$ for a galactic supernova), $E$ is the energy of the neutrinos, $L_{\alpha}$ is the luminosity in the flavor $\nu_{\alpha}$, and $T_{\alpha}$ represents an effective temperature. The normalization factor equals: $F_{3}\left(\eta_{\alpha}\right) \equiv \int_{0}^{\infty} d x x^{3} /\left(e^{x-\eta_{\alpha}}+1\right)$.

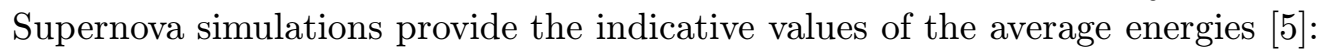

$$
\left\langle E_{\bar{e}}\right\rangle=(14-22) \mathrm{MeV}, \quad\left\langle E_{x}\right\rangle /\left\langle E_{\bar{e}}\right\rangle=(1.1-1.6), \quad\left\langle E_{e}\right\rangle /\left\langle E_{\bar{e}}\right\rangle=(0.5-0.8),
$$

and the typical value of the (time integrated) luminosity in each flavor: $L_{\alpha} \sim(1-5)$. $10^{52}$ ergs. The luminosities of all neutrino species are expected to be approximately equal, within a factor of two or so [i. The $\nu_{\mu}$ and $\nu_{\tau}\left(\bar{\nu}_{\mu}\right.$ and $\left.\bar{\nu}_{\tau}\right)$ spectra are equal with good approximation, and therefore the two species can be treated as a single one, $\nu_{x}\left(\bar{\nu}_{x}\right)$. The pinching parameter $\eta_{\alpha}$ can vary between 0 and $\sim 3$ for $\nu_{e}$ and $\bar{\nu}_{e}$, while smaller pinching is expected for $\nu_{\mu}, \nu_{\tau}: \eta_{\mu}=\eta_{\tau} \sim 0-2$.

The matter density profile met by the neutrinos can be approximated, at least in the first few seconds of their emission, by that of the progenitor star [i़i]. The latter is well described by the radial power law [î́]

$$
\rho(r)=10^{13} C\left(\frac{10 \mathrm{~km}}{r}\right)^{3} \mathrm{~g} \cdot \mathrm{cm}^{-3}
$$

with $C \simeq 1-15$.

\section{Conversion in the star, jump probability and $\theta_{13}$}

Let us consider the conversion of neutrinos as they propagate from the production region outward in the star, for the case of normal mass hierarchy $\left(\Delta m_{32}^{2}>0\right)$. As shown in Fig. (positive density semi-plane), the eigenvalues of the Hamiltonian in matter and the flavor composition of its eigenstates change with the variation of the matter density along the neutrino trajectory.

At production, the mixing is suppressed due to the very large density $\left(\rho \sim 10^{11} \mathrm{~g} \cdot \mathrm{cm}^{-3}\right)$, therefore the eigenstates of the Hamiltonian coincide with the flavor states. At lower densities, the neutrinos undergo two MSW resonances (level-crossings). The inner resonance $(\mathrm{H})$ is governed by the parameters $\Delta m_{32}^{2}$ and $\theta_{13}$ and is realized at $\rho \sim 10^{3} \mathrm{~g} \cdot \mathrm{cm}^{-3}(10 \mathrm{MeV} / E)$. The probability of transition between the eigenstates of the Hamiltonian (jump probability) in this resonance, $P_{H}$, strongly depends on $\theta_{13}$ as discussed later in this section. The second resonance, (L) is determined by $\Delta m_{21}^{2}$ and $\theta_{12}$ and happens at lower density, $\rho \sim(30-140)(10 \mathrm{MeV} / E) \mathrm{g} \cdot \mathrm{cm}^{-3}$. For the values of parameters in Eq. (1.1. probability in this resonance is negligibly small (adiabatic propagation). The neutrinos leave the star as mass eigenstates and therefore do not oscillate on the way from the star to the Earth. If they cross the Earth before detection, oscillations are restarted due to Earth matter effects (see e.g. [i]

Since they have opposite sign of the matter potential, antineutrinos do not undergo any resonance in the matter of the star (negative density semi-plane in Fig. iiij).

As an effect of conversion, the $\nu_{e}$ and $\bar{\nu}_{e}$ fluxes in the detector, $F_{e}$ and $F_{\bar{e}}$, are combinations of the original $\nu_{e}$ and $\nu_{x}\left(\bar{\nu}_{e}\right.$ and $\left.\bar{\nu}_{x}\right)$ fluxes. Considering for simplicity the case of 


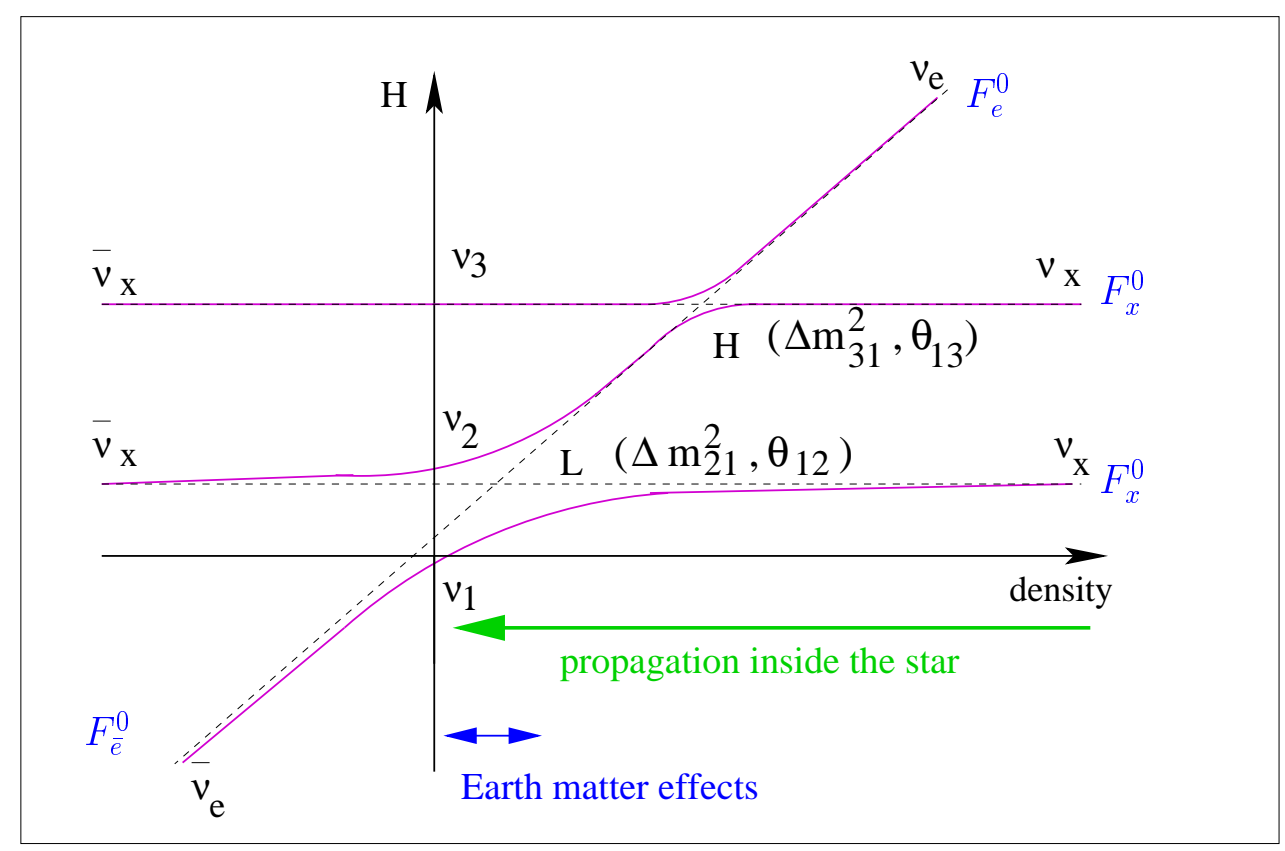

Figure 1: The level-crossing diagram for normal mass hierarchy. The solid curves represent the eigenvalues of the Hamiltonian in matter.

no Earth crossing, one gets:

$$
\begin{aligned}
& F_{e}=P_{H} \sin ^{2} \theta_{12} F_{e}^{0}+\left(1-P_{H} \sin ^{2} \theta_{12}\right) F_{x}^{0}, \\
& F_{\bar{e}}=\cos ^{2} \theta_{12} F_{\bar{e}}^{0}+\sin ^{2} \theta_{12} F_{\bar{x}}^{0} .
\end{aligned}
$$

For inverted hierarchy $\left(\Delta m_{32}^{2}<0\right)$, the $\mathrm{H}$ resonance is in the antineutrino channel, while the $\mathrm{L}$ resonance is unaffected. In this case the fluxes in the detector equal:

$$
\begin{aligned}
& F_{e}=\sin ^{2} \theta_{12} F_{e}^{0}+\cos ^{2} \theta_{12} F_{x}^{0}, \\
& F_{\bar{e}}=P_{H} \cos ^{2} \theta_{12} F_{\bar{e}}^{0}+\left(1-P_{H} \cos ^{2} \theta_{12}\right) F_{\bar{x}}^{0} .
\end{aligned}
$$

As expected, here the jump probability $P_{H}$ appears in the expression of the $\bar{\nu}_{e}$ flux, in

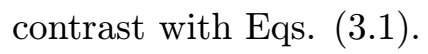

In summary, the supernova neutrino signal is sensitive to the mass hierarchy and to $\theta_{13}$ for the following reasons: (i) depending on the hierarchy, the $\mathrm{H}$ resonance affects either neutrinos or antineutrinos; (ii) the observed $\nu_{e}$ or $\bar{\nu}_{e}$ fluxes depend on the value of $\theta_{13}$ via the jump probability $P_{H}$. The latter can be calculated using the Landau-Zener formula

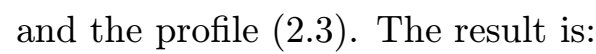

$$
P_{H}=\exp \left[-\left(\frac{1.08 \cdot 10^{7} \mathrm{MeV}}{E}\right)^{2 / 3}\left(\frac{\Delta m_{32}^{2}}{10^{-3} \mathrm{eV}^{2}}\right)^{2 / 3} C^{1 / 3} \sin ^{2} \theta_{13}\right]
$$

It follows that three regions exist: 
(i) Adiabaticity breaking region: $\sin ^{2} \theta_{13} \lesssim 10^{-6}(E / 10 \mathrm{MeV})^{2 / 3}$, where $P_{H} \simeq 1$;

(ii) Transition region: $\sin ^{2} \theta_{13} \sim\left(10^{-6}-10^{-4}\right) \cdot(E / 10 \mathrm{MeV})^{2 / 3}$, where $0 \lesssim P_{H} \lesssim 1$;

(iii) Adiabatic region: $\sin ^{2} \theta_{13} \gtrsim 10^{-4}(E / 10 \mathrm{MeV})^{2 / 3}$, where $P_{H} \simeq 0$.

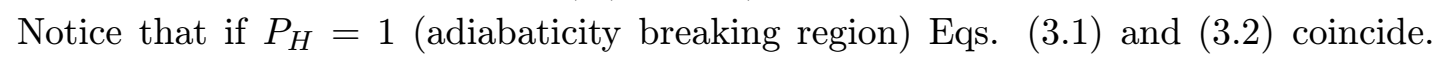
Thus, we get equal predictions for normal and inverted hierarchy and any sensitivity to

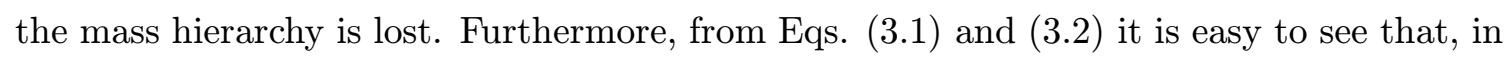
the extreme case in which the original fluxes in the different flavors are equal $\left(F_{\bar{e}}^{0}=F_{\bar{x}}^{0}\right.$, $\left.F_{e}^{0}=F_{x}^{0}\right)$, conversion effects cancel and one has $F_{e}=F_{e}^{0}, F_{\bar{e}}=F_{\bar{e}}^{0}$.

\section{Earth matter effects}

If the neutrinos cross the Earth before detection, they undergo regeneration effects due to the interaction with the matter of the Earth. Indeed, the matter density in the Earth, $\rho \sim(1-13) \mathrm{g} \cdot \mathrm{cm}^{-3}$, is close to the density at the $\mathrm{L}$ resonance in the star (see Sec. $1 \overline{3}$ i), for which the mixing angle $\theta_{12}$ is resonantly enhanced. This implies that the amplitude of neutrino oscillations in the Earth can be significant and lead to observable effects.

The results $\left(\left(\overline{3} \cdot \overline{1}_{1}\right)\right.$ and $(\overline{3} \cdot \overline{2})$ can be immediately generalized by the replacements:

$$
\sin ^{2} \theta_{12} \rightarrow P_{2 e} \quad \cos ^{2} \theta_{12} \rightarrow P_{1 \bar{e}}
$$

where $P_{2 e}\left(P_{1 \bar{e}}\right)$ is the probability that a neutrino (antineutrino) arriving at Earth in the state $\nu_{2}\left(\bar{\nu}_{1}\right)$ is detected as $\nu_{e}\left(\bar{\nu}_{e}\right)$ in the detector. It depends on the oscillation parameters $\theta_{12}$ and $\Delta m_{21}^{2}$, on the Earth density profile, on the neutrino arrival direction and on the neutrino energy. The dependence on the energy has an oscillatory character, resulting in characteristic distortions of the energy spectra of observed events. The distortions are different for detectors at different locations on the planet.

As an example, consider two detectors, D1 and D2, with D2 shielded by the Earth and D1 unshielded. The differences of the $\nu_{e}$ and $\bar{\nu}_{e}$ fluxes in the two detectors follow from

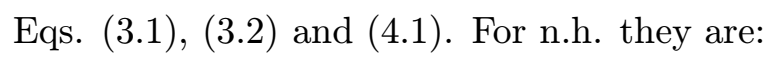

$$
\begin{aligned}
& F_{e}^{D 2}-F_{e}^{D 1}=P_{H}\left(P_{2 e}-\sin ^{2} \theta_{12}\right)\left(F_{e}^{0}-F_{x}^{0}\right), \\
& F_{\bar{e}}^{D 2}-F_{\bar{e}}^{D 1}=\left(P_{1 \bar{e}}-\cos ^{2} \theta_{12}\right)\left(F_{\bar{e}}^{0}-F_{\bar{x}}^{0}\right),
\end{aligned}
$$

while for i.h. one gets:

$$
\begin{aligned}
& F_{e}^{D 2}-F_{e}^{D 1}=\left(P_{2 e}-\sin ^{2} \theta_{12}\right)\left(F_{e}^{0}-F_{x}^{0}\right), \\
& F_{\bar{e}}^{D 2}-F_{\bar{e}}^{D 1}=P_{H}\left(P_{1 \bar{e}}-\cos ^{2} \theta_{12}\right)\left(F_{\bar{e}}^{0}-F_{\bar{x}}^{0}\right) .
\end{aligned}
$$

The dependence of these differences on the neutrino mass hierarchy and on $\theta_{13}$ has the same origin as that of conversion effects in the star and can be described in analogous terms (Sec. $\left.\overline{\beta_{1}}\right)$.

Figure ${ }_{2}^{2}$ gives an illustration of the energy spectra of events predicted from Eq. (4.2.2) for two identical water Cerenkov detectors. 
$\mathrm{dN} / \mathrm{dE}$

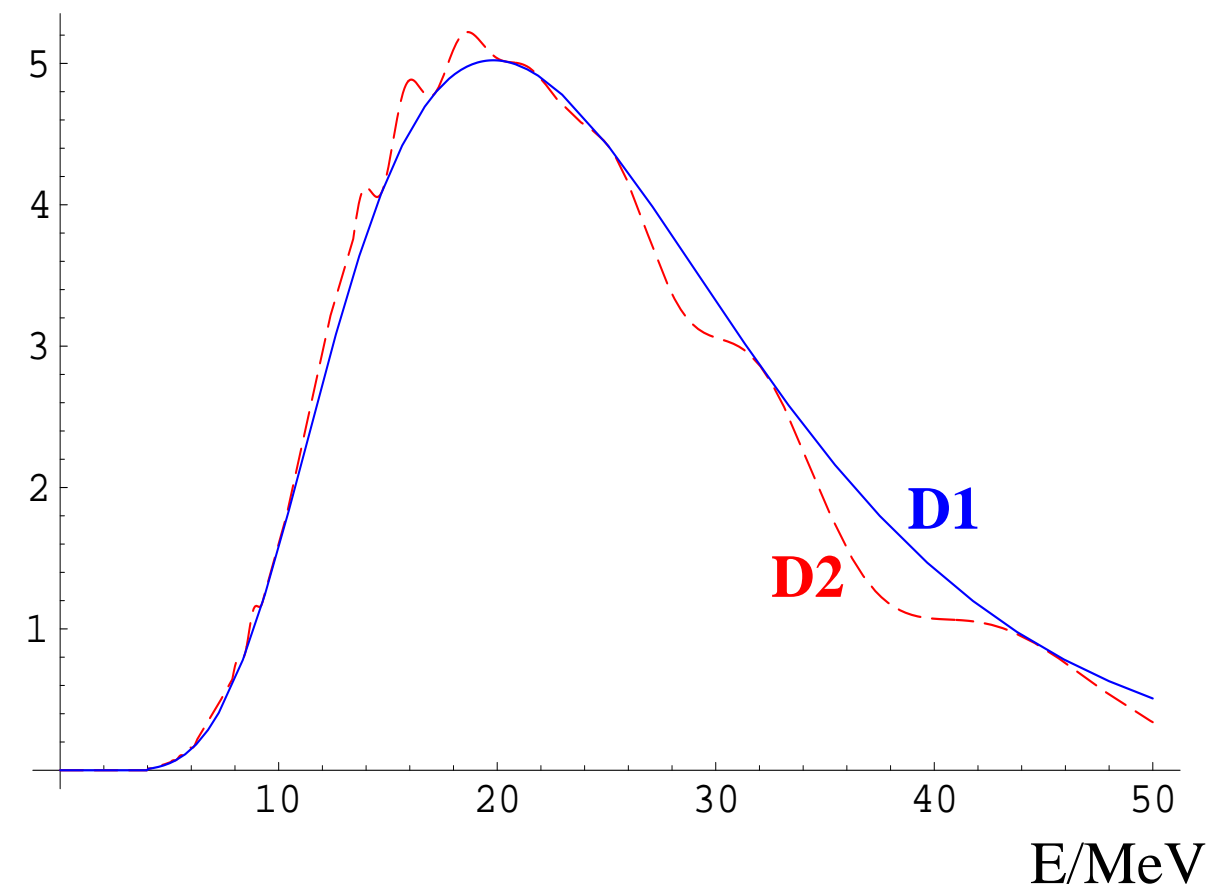

Figure 2: Predicted energy spectra of positrons (arbitrary units on the vertical axis) from the $\bar{\nu}_{e}+p \rightarrow n+e^{+}$reaction at two identical water Cerenkov detectors, one of which (D2) is shielded by the Earth with nadir angle $\theta_{n}=70^{\circ}$. I used the detection efficiency of the SuperKamiokande detector and the parameters: $T_{\bar{e}}=5 \mathrm{MeV}, T_{\bar{x}}=7 \mathrm{MeV}$, normal hierarchy (or i.h. with $P_{H}=1$ ) and $\Delta m_{21}^{2}=7 \cdot 10^{-5} \mathrm{eV}^{2}$. Equal luminosities have been taken in $\bar{\nu}_{e}$ and $\bar{\nu}_{x}$ and the detailed Earth density profile from ref.

\section{Probing $\theta_{13}$ and the mass hierarchy}

There are several approaches to probe the neutrino oscillation parameters and at the same time take into account the uncertainties on the features of the original fluxes:

1. to perform a global fit of the data, determining both the oscillation parameters and the parameters of the original fluxes simultaneously $\left.\left[\bar{p}_{1}^{1}, 1\right] \underline{1} \underline{0}\right]$. With this method a completely general analysis is not possible due to the large number of parameters involved.

2. to single out and study (numerically and analytically) specific observables which (i) have maximal sensitivity to the oscillation parameters of interest and (ii) whose dependence on the astrophysical uncertainties is minimal or well understood [i] $\left.\overline{1}_{-1}^{1}\right]$.

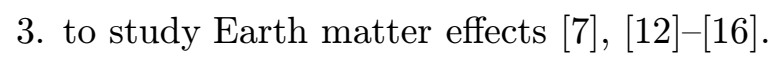

4. to study the effects of shock-wave propagation on the neutrino signal. The shockwave driving the supernova explosion modifies the density profile of the star at the resonance points, thus changing the conversion pattern inside the star and the observed neutrino

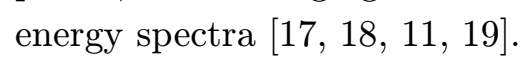

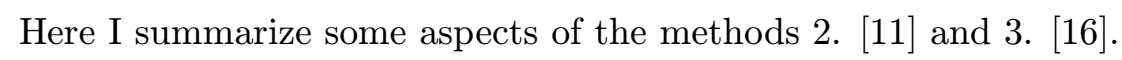




\subsection{Observables}

Let us consider the method of the observables (item 2.). A good prescription to find observables which fit the criteria of sensitivity and uncertainty-freedom stated above ((i) and (ii)) is to consider the parameters describing the energy spectra of events induced by $\nu_{e}$, and the same parameters for the $\bar{\nu}_{e}$-induced spectra, and take their ratios.

For instance, let us consider the spectra of $\nu_{e}$ events at the SNO detector from the CC scattering on deuterium, $\nu_{e}+d \rightarrow p+p+e^{-}$, and the $\bar{\nu}_{e}$ events at the SuperKamiokande detector from inverse beta decay, $\bar{\nu}_{e}+p \rightarrow n+e^{+}$.

We can define the following four observables: (1) the ratio of the average energies, $r_{E}$, and (2) the ratio of the widths, $r_{\Gamma}$, of the $\nu_{e}$ and $\bar{\nu}_{e}$-induced spectra:

$$
r_{E}=\frac{\langle E\rangle}{\langle\bar{E}\rangle}, \quad r_{\Gamma} \equiv \frac{\Gamma}{\bar{\Gamma}}
$$

(3) the ratios of the numbers of $\nu_{e}$ and $\bar{\nu}_{e}$ events in the low energy tails, $S$, and (4) in the high energy tails, $R_{\text {tail }}$ :

$$
S \equiv \frac{N_{e}\left(E<E_{L}^{\prime}\right)}{N_{\bar{e}}\left(E<\bar{E}_{L}^{\prime}\right)}, \quad R_{\text {tail }}\left(E_{L}, \bar{E}_{L}\right) \equiv \frac{N_{e}\left(E>E_{L}\right)}{N_{\bar{e}}\left(E>\bar{E}_{L}\right)} .
$$

Here the overbarred quantities refer to antineutrino spectra, and the width $\Gamma$ is defined as $\Gamma \equiv\left(\left\langle E^{2}\right\rangle /\langle E\rangle^{2}-1\right)^{1 / 2}$. The high and low energy cuts, $E_{L}, \bar{E}_{L}, E_{L}^{\prime}, \bar{E}_{L}^{\prime}$ can be suitably chosen to optimize the analysis [i] ${ }_{1}^{1} \overline{1}_{1}$.

\subsection{Distinguishing between extreme possibilities: scatter plots}

Let us consider the three extreme cases:

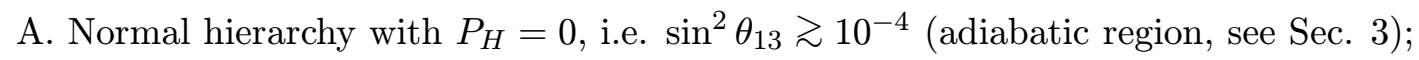

B. Inverted hierarchy with $P_{H}=0$;

C. $P_{H}=1$, corresponding to $\sin ^{2} \theta_{13} \lesssim 10^{-6}$, with normal or inverted hierarchy (recall that results do not depend on the hierarchy in this case, see Sec.

Figure $13 \overline{3}$ ' shows the regions in the space of the observables $r_{E}, r_{\Gamma}, R_{\text {tail }}$ for the cases A, B, C, obtained by scanning over the astrophysical parameters in the intervals discussed in Sec. 2 . The values of the oscillation parameters $\left|\Delta m_{32}^{2}\right|, \Delta m_{21}^{2}, \theta_{23}$ and $\theta_{12}$ have been taken to coincide with the current best fit points with $10 \%$ error, as expected from near future measurements. To calculate $R_{\text {tail }}$ the cuts $E_{L}=45 \mathrm{MeV}$ and $\bar{E}_{L}=55$ have been used.

The results in the figure can be easily interpreted in terms of the different size of the conversion effects in the different cases [i] $\left.{ }_{1}^{1} \overline{1}\right]$. They show that large regions of the parameter space exist where only one among the scenarios A, B or C is possible. Also regions appear where two of these scenarios are realized. If these regions are selected by the experiments, the third possibility will be excluded.

The scenarios in which $0<P_{H}<1$ are not shown in Fig. $\underline{3}_{-1}$ For normal hierarchy and $0<P_{H}<1$ we expect the allowed region to be intermediate between the regions found for A and C. Similarly, for inverted hierarchy and $0<P_{H}<1$ the region of possible 

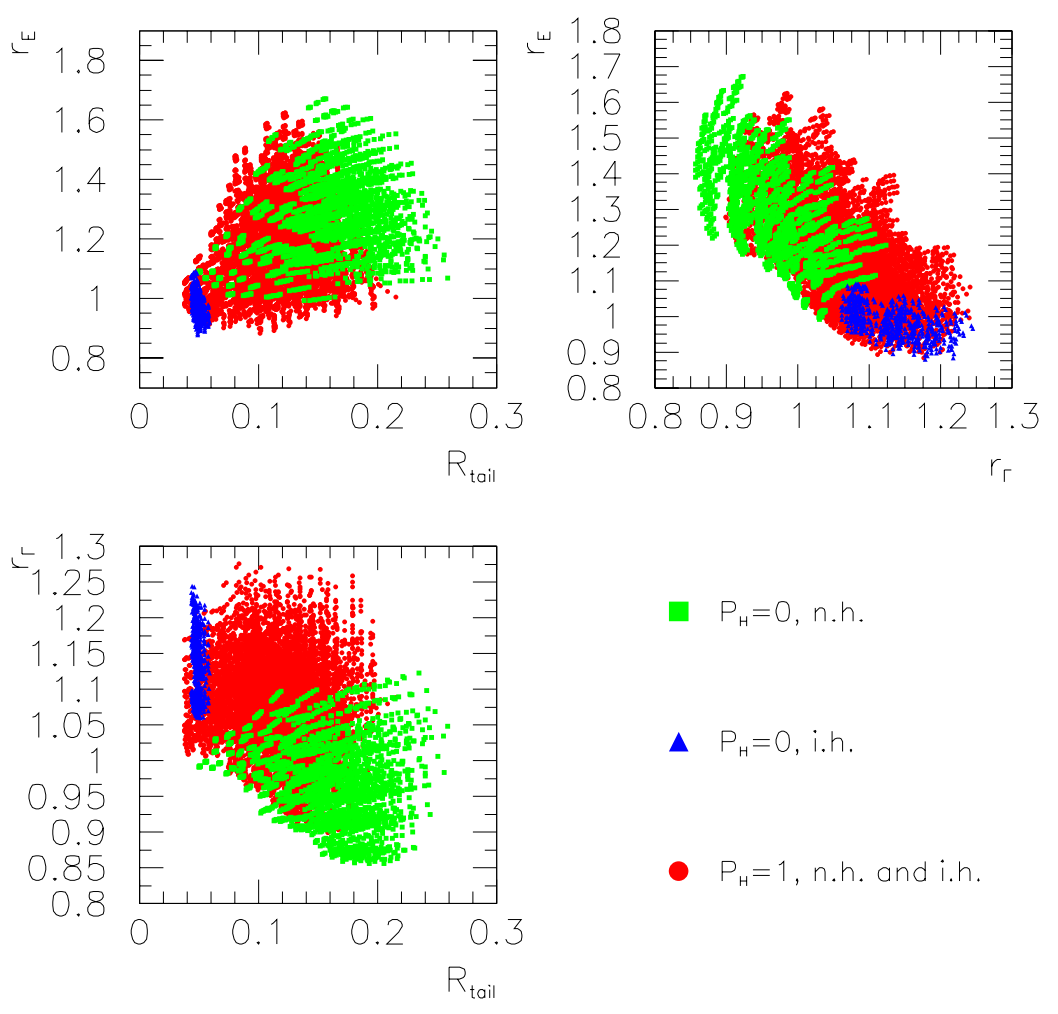

Figure 3: Scatter plot in the space of the observables $r_{E}, r_{\Gamma}, R_{\text {tail }}$ for the cases A, B, C discussed in the text.

values of parameters is intermediate between the regions of cases B and C. For this reason, the conclusions I derived from Fig. character of establishing one of the scenarios $\mathrm{A}, \mathrm{B}, \mathrm{C}$.

It is clear that the potential of the method I have discussed here depends on the statistics and therefore on the distance from the supernova. It can be checked [i] a relatively close star $(D \lesssim 4 \mathrm{kpc})$ the error bars are substantially smaller than the field of points so that the discrimination of different cases is possible.

\subsection{Analyzing the Earth matter effects}

The study of Earth regeneration effects is particularly promising. The reason is that the information on $\theta_{13}$ and on the mass hierarchy which can be obtained by this method is largely independent of astrophysical uncertainties.

This can be understood considering that:

- The main signature of Earth matter effects - consisting in oscillatory modulations of the observed energy spectra (see Sec. $\overline{4}$ i $)$ - is unambiguous since it can not be 
mimicked by any astrophysical phenomenon. Moreover the pattern of oscillation minima and maxima depends only on $\Delta m_{21}^{2}$ and $\theta_{12}$, which probably will be known precisely from solar neutrino experiments and KamLand before the next galactic supernova event. This will improve the possibility of identification of Earth matter effects.

- To obtain unambiguous conclusions on $\theta_{13}$ and on the mass hierarchy it is enough that experiments exclude or establish the Earth matter effect, without measuring its size precisely, especially if the effect is probed in both the $\nu_{e}$ and $\bar{\nu}_{e}$ channels. The fact that precision is not necessary is very important in this specific problem, where large astrophysical uncertainties are present.

To illustrate the latter point in more detail (see also Table the following scenario.

(i) At the time of arrival of the neutrino burst, at least one running detector is shielded by the Earth.

(ii) The shielded detectors record data due to both $\nu_{e}$ and $\bar{\nu}_{e}$. The two $\left(\nu_{e}\right.$ and $\left.\bar{\nu}_{e}\right)$ sets of events can be efficiently separated and for each of them the energy spectrum of the incoming neutrinos can be reconstructed with good resolution.

(iii) The statistics of both the data sets are sufficiently large so that (oscillatory) spectral distortions as large as $\sim 10-20 \%$ can be established with high statistical significance.

If these conditions are fulfilled, we have four possible experimental results. The corresponding conclusions on $\theta_{13}$ and on the neutrino mass hierarchy are discussed below and

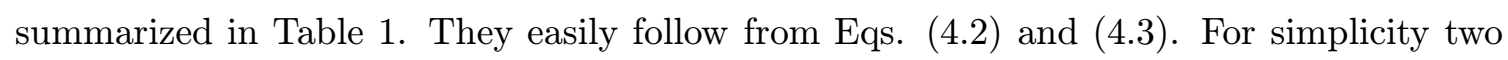
possibilities are considered in the discussion. The first is the case in which substantial differences in the fluxes of different flavors are assumed, on the basis of (future) precise theoretical predictions. In the second case astrophysical uncertainties are large and allow equality of the original fluxes. The generalization of the discussion to intermediate cases is straightforward.

1. The Earth effects are established in both $\nu_{e}$ and $\bar{\nu}_{e}$ channel. In this case unique conclusions are obtained on the oscillation parameters and on the original fluxes at the same time. A first result is the difference of the original fluxes in the different flavors: $F_{e}^{0} \neq F_{x}^{0}$ and $F_{\bar{e}}^{0} \neq F_{\bar{x}}^{0}$. This would be an important test of calculations of neutrino spectra formation inside the star. Secondly, we get that $P_{H}$ is significantly different from zero, $P_{H} \sim 1$. This gives the upper bound $\sin ^{2} \theta_{13} \lesssim 10^{-6}$ (see Sec. $\underline{3}_{-1}$ ). The mass hierarchy remains undetermined.

2. The Earth effect is seen in the $\nu_{e}$ channel only, and excluded in the $\bar{\nu}_{e}$ channel. This tells us that $F_{e}^{0} \neq F_{x}^{0}$. If $F_{\bar{e}}^{0} \neq F_{\bar{x}}^{0}$ is assumed, again the conclusion is unambiguous: the mass hierarchy is inverted and $P_{H} \sim 0$, corresponding to $\sin ^{2} \theta_{13} \gtrsim 10^{-4}$. In absence of a priori assumptions on the fluxes, we must consider that the equality $F_{\bar{e}}^{0} \simeq F_{\bar{x}}^{0}$ could suppress the Earth matter effect on antineutrinos, allowing other scenarios of hierarchy and $\theta_{13}$. Nevertheless, the case of normal hierarchy with $P_{H} \sim 0$ remains excluded. 
3. The Earth effect is established in the $\bar{\nu}_{e}$ channel only, while excluded in the $\nu_{e}$ channel. Similarly to the previous case, here we conclude that $F_{\bar{e}}^{0} \neq F_{\bar{x}}^{0}$. Assuming that the neutrino original fluxes are different, we have that the normal hierarchy is singled out and $P_{H} \sim 0\left(\sin ^{2} \theta_{13} \gtrsim 10^{-4}\right)$. The possibility that $F_{e}^{0} \simeq F_{x}^{0}$ appears exotic, since both numerical calculations and simple physical considerations predict that the difference $\left(F_{x}^{0}-F_{e}^{0}\right)$ should be larger than $\left(F_{\bar{x}}^{0}-F_{\bar{e}}^{0}\right)$. If the case $F_{e}^{0} \simeq F_{x}^{0}$ is allowed, still the scenario of inverted hierarchy with $P_{H} \sim 0$ is excluded.

4. No Earth effect is seen in both $\nu_{e}$ and $\bar{\nu}_{e}$ channel. As can be easily realized, this result requires that at least in one channel $\left(\nu_{e}\right.$ or $\left.\bar{\nu}_{e}\right)$ oscillations are suppressed by equality of original fluxes: $F_{e}^{0} \simeq F_{x}^{0}$ or $F_{\bar{e}}^{0} \simeq F_{\bar{x}}^{0}$. This would be interesting as a test of predictions of the neutrino fluxes and energy spectra. No definite conclusions on oscillation parameters are possible.

\begin{tabular}{ccccc}
\hline \hline$\nu_{e}$ & $\bar{\nu}_{e}$ & CONCLUSIONS: & \\
& & fluxes & hierarchy & $P_{H}\left(\sin ^{2} \theta_{13}\right)$ \\
\hline \hline yes & yes & different & undetermined & $P_{H} \sim 1\left(\sin ^{2} \theta_{13} \lesssim 10^{-6}\right)$. \\
\hline yes & no & $F_{e}^{0} \neq F_{x}^{0}$ and $F_{\bar{e}}^{0} \neq F_{\bar{x}}^{0}$ & inverted & $P_{H} \sim 0\left(\sin ^{2} \theta_{13} \gtrsim 10^{-4}\right)$ \\
& & & & \\
& & $F_{e}^{0} \neq F_{x}^{0}$ and $F_{\bar{e}}^{0} \simeq F_{\bar{x}}^{0}$ & exclusion of normal & hierarchy with $P_{H} \sim 0$ \\
\hline no & yes & different fluxes & normal & $P_{H} \sim 0\left(\sin ^{2} \theta_{13} \gtrsim 10^{-4}\right)$ \\
\hline no & no & $F_{e}^{0} \simeq F_{x}^{0}$ or $F_{\bar{e}}^{0} \simeq F_{\bar{x}}^{0}$ & any of the above & any of the above \\
\hline \hline
\end{tabular}

Table 1: Summary of conclusions that can be drawn from different cases of observation ("yes") or exclusion ("no") of Earth matter effects in $\nu_{e}$ and $\bar{\nu}_{e}$ channels.

The discussion can be generalized to different experimental setups. For instance, let us consider the situation in which the Earth matter effect is probed in the $\bar{\nu}_{e}$ channel only (data on $\nu_{e}$ may not be available or the $\nu_{e}$ detector is not shielded by the Earth). If the effect is seen, we can exclude the combination of inverted hierarchy and $P_{H} \sim 0$. If it is not, the same combination is established provided that differences in the original fluxes are assumed from theory. If the equality of original fluxes is allowed, no conclusions are possible (see Table $\left.i_{-1}^{\prime}\right)$. If the Earth matter effects are probed in the $\nu_{e}$ channel only, a similar argument applies, leading to the exclusion or establishment of the combination $P_{H} \sim 0$ with normal hierarchy. Again, results depend on the size of the uncertainties in the original neutrino fluxes.

\subsection{Remarks on the Earth matter effect}

Interesting aspects emerge from the discussion in Sec.

One of them is the importance of combining different data sets. Probing the Earth matter effects in both the $\nu_{e}$ and the $\bar{\nu}_{e}$ channel is crucial to disentangle the information on the 1-3 mixing and on the mass hierarchy from astrophysical uncertainties. If the Earth 
regeneration effect is probed in one channel only the conclusions on the mass hierarchy and $\theta_{13}$, though potentially strong, have only a "conditional" character, since they depend on assumptions on the original neutrino fluxes.

A second, very important, point is that a negative result is stronger than a positive one. Indeed, if the Earth effect is seen on both neutrinos and antineutrinos (case 1. of Sec. (5. of the Earth matter effect in $\nu_{e}$ channel, together with a positive result on $\bar{\nu}_{e}$ (case 3.), one obtains both a lower bound on $\theta_{13}$ and establishes the normal mass hierarchy. The inverted hierarchy is excluded for any value of $\theta_{13}$. Indeed, as shown in Sec. '3in', for inverted hierarchy the $\mathrm{H}$ resonance is in the antineutrino channel. In the neutrino channel nothing prevents the transition, inside the star, of $\nu_{e}$ to $\nu_{2}$, which then should oscillate in the matter of the Earth. So one should see the Earth matter effect in the neutrino channel.

Remarkably, the same conclusion holds even if the negative result for $\nu_{e}$ is the only information available, provided that the inequality of original fluxes can be safely assumed from theory. A similar argument is valid for the case of non observation in the $\bar{\nu}_{e}$ channel, with the cautionary remark that $F_{\bar{e}}^{0} \simeq F_{\bar{x}}^{0}$ could be realized and lead to weaker conclusions.

It should be also considered that a negative result on the Earth regeneration effect in one of the channels could be an indication of the existence of a fourth, sterile, neutrino species. The neutrino mass spectrum would have the so called $(3+1)$ form, characterized by a strong hierarchy between the fourth mass eigenstate, predominantly sterile in flavor,

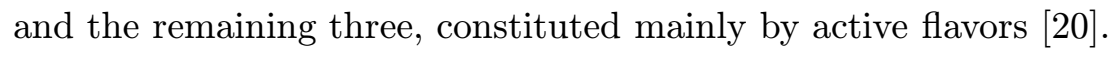

In the event that the study of Earth matter effects is not conclusive - on the type of neutrino mass spectrum and on the 1-3 mixing - due to uncertainties in the original neutrino fluxes, the remaining ambiguities could be resolved by the combination with other observations or by the study of specific features of the Earth effect itself. For instance, the adiabatic region, $\sin ^{2} \theta_{13} \gtrsim 10^{-4}$, could be selected if the Earth matter effect is initially absent and appears only at late times due to shock-wave effects [i]1i]. The channel in which this happens would also determine the mass hierarchy.

Shock-wave effects could also confirm or exclude the presence of a sterile neutrino, together with other results like, e.g., the absence of the early peak in the $\nu_{e}$ luminosity

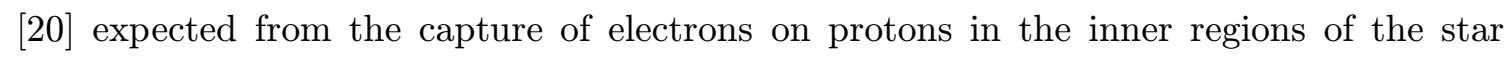
(neutronization peak).

\section{Acknowledgments}

I am grateful to the organizers and the participants of AHEP2003 for the stimulating atmosphere I enjoyed there. The research work presented in these proceedings was supported by the Keck fellowship and the NSF grants PHY-0070928 and PHY99-07949. My participation to the AHEP2003 conference was made possible by a partial support from the organizing committee and by funding from IAS. 


\section{References}

[1] See e.g. the review by C. Giunti, hep-ph/0305139, for a summary and references.

[2] See e.g. the analysis and references in G. L. Fogli, E. Lisi, A. Marrone and D. Montanino, Phys. Rev. D67, 093006 (2003), hep-ph/0303064.

[3] CHOOZ Collaboration, M. Apollonio et. al., Phys. Lett. B466 (1999) 415-430.

[4] F. Boehm et. al., Phys. Rev. D62 (2000) 072002.

[5] See e.g. M. T. Keil, G. G. Raffelt, and H.-T. Janka, Astrophys. J. 590:971-991, 2003, astro-ph/0208035, and references therein.

[6] G. E. Brown, H. A. Bethe and G. Baym, Nucl. Phys. A 375 (1982) 481.

[7] A. S. Dighe and A. Y. Smirnov, Phys. Rev. D62 (2000) 033007.

[8] A. M. Dzewonski and D. L. Anderson, Phys. Earth. Planet. Inter. 25 (1981) 297.

[9] V. Barger, D. Marfatia, and B. P. Wood, Phys. Lett. B547 (2002) 37-42.

[10] H. Minakata, H. Nunokawa, R. Tomas, and J. W. F. Valle, Phys. Lett. B542 (2002) 239-244.

[11] C. Lunardini and A. Y. Smirnov, JCAP 0306:009 (2003), hep-ph/0302033.

[12] C. Lunardini and A. Y. Smirnov, Nucl. Phys. B 616, 307 (2001), hep-ph/0106149.

[13] K. Takahashi and K. Sato, Phys. Rev. D 66, 033006 (2002), hep-ph/0110105.

[14] A. S. Dighe, M. T. Keil and G. G. Raffelt, JCAP 0306:005 (2003), hep-ph/0303210.

[15] A. S. Dighe, M. T. Keil and G. G. Raffelt, JCAP 0306:006 (2003), hep-ph/0304150.

[16] The present discussion is based on the paper by C. Lunardini and A. Y. Smirnov, in preparation.

[17] R. C. Schirato, G. M. Fuller, astro-ph/0205390.

[18] K. Takahashi, K. Sato, H. E. Dalhed and J. R. Wilson, astro-ph/0212195.

[19] G. L. Fogli, E. Lisi, D. Montanino and A. Mirizzi, Phys. Rev. D 68033005 (2003), hep-ph/0304056.

[20] The expected supernova neutrino signal is discussed in O. L. Peres and A. Y. Smirnov, Nucl. Phys. B 599, 3 (2001), hep-ph/0011054.

[21] See e.g. C. Waltham, proceedings from the International Conference on Cosmic Rays (ICRC) 2001, available at www.copernicus.org/icrc/papers/ici7144_p.pdf .

[22] D. B. Cline, F. Sergiampietri, J. G. Learned and K. McDonald, Nucl. Instrum. Meth. A 503, 136 (2003), astro-ph/0105442.

[23] A. Bueno, I. Gil-Botella and A. Rubbia, hep-ph/0307222.

[24] J. F. Beacom and M. R. Vagins, in preparation; see e.g. the talk by M. R. Vagins at the NOON 2003 conference, Kanazawa, Japan, February 4th -10th, available at http://www-sk.icrr.u-tokyo.ac.jp/noon2003/.

[25] S. R. Elliott, Phys. Rev. C 62, 065802 (2000), astro-ph/0006041.

[26] J. Engel, G. C. McLaughlin and C. Volpe, Phys. Rev. D 67, 013005 (2003), hep-ph/0209267.

[27] C. J. Horowitz, K. J. Coakley and D. N. McKinsey, Phys. Rev. D 68023005 (2003), astro-ph/0302071. 